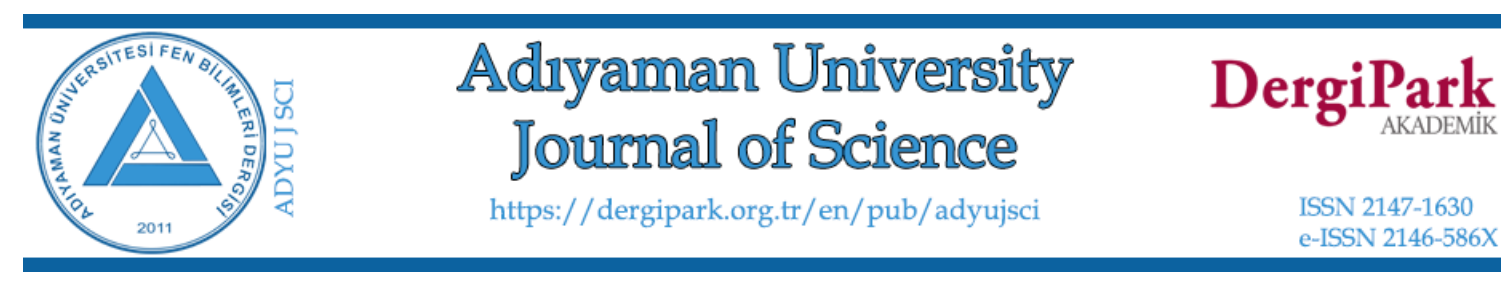

\title{
Gall Forming Wasp Species of Oak Forests in Bolu
}

\author{
Serap MUTUN ${ }^{1}$, Serdar DİNÇ ${ }^{1, *}$ \\ ${ }^{1}$ Bolu Abant Izzet Baysal University, Faculty of Science and Art, Department of Biology, Bolu, Turkey \\ smutun@ibu.edu.tr,ORCID:0000-0002-0838-3857 \\ dinc_s@ibu.edu.tr,ORCID:0000-0002-8920-2738
}

\begin{abstract}
Oak cynipid galls were collected between 2006-2019 to reveal species diversity of oak gall wasps (Hymenoptera, Cynipidae, Cynipini) in Bolu. Collected galls were brought to laboratory and kept at room temperature in jars covered by tulle until adult cynipid wasps emerged. As a result, 44 species from 8 genera showed that Andricus is the most specious genus with 26 species. Our results indicate that Bolu bears high gall wasp species diversity. In this study, new distribution ranges are provided for most of the cynipid species previously reported for Turkey.
\end{abstract}

Keywords: Cynipidae; Hymenoptera; Oak gall wasp; Diversity; Bolu.

\section{Bolu Meşe Ormanlarında Gal Oluşturan Arı Türleri}

\section{Öz}

Meşe gal arısı (Hymenoptera, Cynipidae, Cynipini) çeşitliliğini belirleyebilmek için 20062019 yılları arasında Bolu'da arazi çalışmaları yapılmıştır. Toplanan mazılar laboratuvara getirilerek kavanozlara konulmuş ve tülle kapatılarak oda sıcaklığında ergin gal arısı elde edilene dek tutulmuşlardır. Çalışma sonucunda belirlenen 8 cinse ait toplam 44 cynipid türü belirlenmiş, 26 tür ile Andricus cinsinin en fazla tür sayısına sahip grup olduğu belirlenmiştir. Sonuçlar 
Bolu'da gal arısı çeşitliliğinin yüksek olduğunu ortaya koymuştur. Bu çalışma ile Türkiye'nin meşe gal arısı türleri için yeni lokasyonlar belirlenmiştir.

Anahtar Kelimeler: Cynipidae; Hymenoptera; Meşe mazı arısı; Çeşitlilik; Bolu.

\section{Introduction}

Different arthropod groups from insects to acaroid species and other organisms from bacteria, fungi to viruses induce galls on a variety of plant taxa [1]. Among insects, the majority of gall-inducing species are gall wasps (Hymenoptera, Cynipidae, Cynipini) that are represented by more than 1400 species classified under 40 genera with worldwide distribution [2]. In the northern hemisphere, the Nearctic region with nearly 700 cynipid species is richer than the Palearctic region, which hosts roughly around 300 species [3, 4]. Gall forming cynipids prefer different parts of predominantly oak species from the family Fagaceae as their hosts [5]. They form their galls in roots, leaves, shoots, branches, stems, flowers, and other parts of oak trees [6].

At the junction of three phytogeographic regions, 24 taxa including species, subspecies and varieties are known to exist in nearly 5.938 .527 hectares area in the Turkish forests [7, 8]. Until recent years, there have not been many studies in oak gall wasps despite the richness in the host plant species in Turkey. However, there is currently an increase in these obligate guests of oaks [9-18]. A recent checklist study reported 148 species under 25 genera of cynipids representing 9 tribes [19]. The tribe Cynipini hosts 110 species under 12 genera and galls variety of oak taxa from Quercus, Cerris and Ilex sections. Majority of the species are from the genus Andricus with 77 species followed by Cynips, Neuroterus and other genera. A recent study added two new records for the Turkish cynipid fauna [20]. Despite the acceleration in oak gall wasp research the Turkish oak cynipid gall wasp fauna still remains little-known, and it is estimated that the number of gall wasp species is much higher than the current reports.

With this study, we aim to contribute to the knowledge of the Turkish oak cynipid fauna with special emphasis given to Bolu since it is rich in oaks. Bolu is located in the northwestern part of Turkey which is an important city being in the passageway between Anatolia and the Thrace considering its floral elements. Among a variety of oak species and their natural hybrids Quercus petraea (Mattuschka) Liebl., Q. robur L. ve Q. pubescens Willd. are the dominant oak species in the forestry areas of Bolu. This paper gives species diversity as well as new distribution localities of some Turkish oak gall wasp species since so far, no study was carried out to determine oak gall wasp fauna of the studied area. 


\section{Materials and Methods}

The materials examined in this study were collected between May and November from 2006 to 2019 from Bolu Province located in the western part of the Black Sea area of Turkey. Oak cynipid galls were collected from Quercus petraea, Q. pubescens, Q. robur and Q. infectoria. Coordinates of the sampling sites were recorded using a Garmin model Geographic Positioning System (GPS). After collecting galls, they were put in a plastic bag and brought into the laboratory. All gall samples were kept in jars covered by tulle under laboratory conditions until cynipid wasps emerged from the galls. Both gall and adult gall wasp morphologies were used for the identification of specimens by the authors following the key provided in Melika 2006 [6]. The materials are kept in Abant Izzet Baysal University, Department of Biology, Molecular Zoology Laboratory, Bolu.

\section{Results and Discussion}

In this study, a total of 44 species under 8 genera (listed below) from the tribe Cynipini were recorded from Bolu.

\section{Andricus aries (Giraud, 1859)}

Material examined: Gerede Dağkara Village, N 40 $47^{\prime}$ E $32^{\circ} 22^{\prime}$, 09-07-2008, 1 gall,

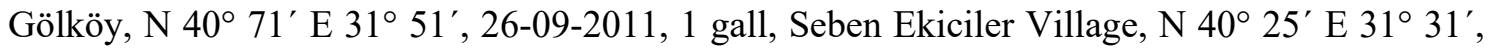
09-07-2008, 2 galls, Güneyce Village, N 40 21' E 31 ${ }^{\circ} 28^{\prime}$, 09-07-2008, 3 galls, Kazanuçuran, N $40^{\circ} 28^{\prime}$ E $31^{\circ} 32^{\prime}$, 09-07-2008, 1 gall. Host: Quercus pubescens, Q. petraea.

\section{Andricus bulgaricus Vassileva-Samnalieva, 1977}

Materyal examined: Gölköy, N 40 $71^{\prime}$ E 31ํ 51', 14-10-2011, 1 gall, Mengen Gökçesu

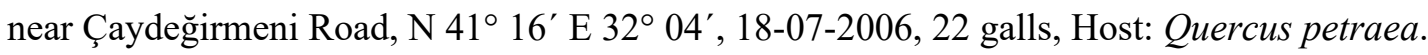

\section{Andricus caputmedusae (Hartig, 1843)}

Material examined: Gölköy, N 40 71' E 31ํ 51', 17-09-2011, 3 galls, Göynük Çamlıca Village, N $40^{\circ} 13$ E $30^{\circ} 56^{\prime}$, 19-08-2010, 5 galls, Kumcuk Village, N $40^{\circ} 22^{\prime}$ E $30^{\circ} 43^{\prime}$, 19-082010, 6 galls, Kürnuç Village, N 40 13’ E 30 41', 19-08-2007, 4 galls, Kıbrıscik Belen Village, $\mathrm{N} 40^{\circ} 22^{\prime}$ E $31^{\circ} 70^{\prime}, 15-07-2009,7$ galls, Kilkara Village, N $40^{\circ} 24^{\prime}$ E $31^{\circ} 46^{\prime}, 15-07-2009,3$ galls. Host: Quercus petraea, Q. infectoria. 


\section{Andricus coriarius (Hartig, 1843)}

Material examined: Gölköy, N 40 $71^{\prime}$ E $31^{\circ}$ 51', 12-09-2011, 4 galls, Göynük Karacalar Village, N 40 $22^{\prime}$ E $30^{\circ} 37^{\prime}$, 19-08-2010, 2 galls, Ahmetbeyler Village, N 40 $12^{\prime}$ E 30 50', 1908-2010, 3 galls, Mengen Çubuk Village, N 40 59’ E 32 04', 14-10-2011, 2 galls, Karakaya village, N 40 $54^{\prime}$ E $31^{\circ}$ 57', 05-08-2009, 2 galls. Host: Quercus petraea.

\section{Andricus coronatus (Giraud, 1859)}

Material examined: Dörtdivan Çetikören Village, N 40 41' E 31 59', 27-07-2006, 7 galls,

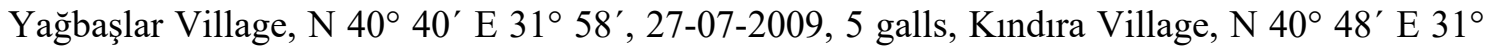
58', 27-07-2006, 6 galls, Gölköy, N 40 71' E 31 51', 14-10-2011, 17 galls, Göynük Arıkçayırı Village, N 40 $28^{\prime}$ E $30^{\circ} 54^{\prime}$, 19-08-2009, 9 galls, Mengen Babahızır Village, N 40 54' E $32^{\circ}$ 03', 14-07-2007, 8 galls, Çubuk Village, N 40 59’ E 32 04', 14-07-2007, 4 galls. Host: Quercus petraea.

\section{Andricus corruptrix (Schechtendal, 1870)}

Material examined: Gölköy, N 40 $71^{\prime}$ E $31^{\circ}$ 51', 01-10-2010, 7 galls, Hamidiye area, N

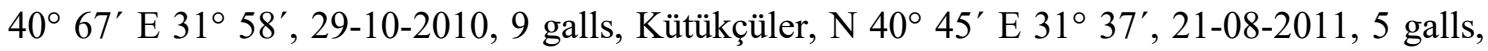
Tokmaklar, N 40 48' E 31 46', 21-08-2011, 8 galls. Host: Quercus petraea, Q. pubescens.

\section{Andricus curvator Hartig, 1840}

Material examined: Gerede Aktaşkurtlar, N 40 50' E 32 31', 22-07-2009, 1 gall, Macarlar

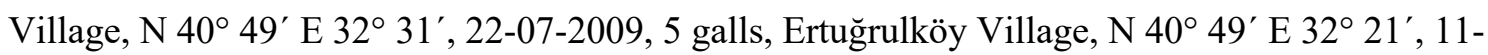
06-2011, 3 galls, Salur-Karapazar, 40 47’ E 32 22', 11-06-2011, 5 galls, Gölköy, N 40 $71^{\prime}$ E $31^{\circ} 51^{\prime}, 11-06-2011,2$ galls, Kıbrıscık near Alan Village, N 40 22' E 31 $45^{\circ}, 13$ galls, Geriş $\mathrm{N}$ $40^{\circ} 22^{\prime}$ E $31^{\circ} 47^{\prime}$, 11-06-2011, 3 galls, Yeniçağa Saray-Yamanlar, N 40 $47^{\prime}$ E $32^{\circ} 22^{\prime}$, 11-062011, 1 gall. Host: Quercus petraea, Q. pubescens.

\section{Andricus foecundatrix (Hartig, 1840)}

Material examined: Dörtdivan Çetikören, N 40 $39^{\prime}$ E $31^{\circ}$ 59', 27-07-2006, 2 galls, Kındıra Village, $\mathrm{N} 40^{\circ} 48^{\prime}$ E $31^{\circ} 50^{\prime}$, 27-07-2007, 1 gall, Yağbaşlar Vilage, N 40 39' E 31 ${ }^{\circ} 59^{\prime}$, 27-072009, 2 galls, Gölköy, N 40 71' E 31 51', 12-09-2011, 6 galls, Kıbrıscık Kılkara Village, N $40^{\circ}$ $24^{\prime}$ E $31^{\circ} 46^{\prime}, 15-07-2009,3$ galls, near Belen Village, N 40 $25^{\prime}$ E $31^{\circ} 45^{\prime}$, 17-07-2009, 3 galls. Host: Quercus petraea, Q. robur. 


\section{Andricus gallaeurnaeformis (Fonscolombe, 1832)}

Material examined: Near Gölköy, N 40 $71^{\prime}$ E 31 51', 10-10-2019, 11 galls, Mudurnu Ekinören Village, N 40 $33^{\prime}$ E $31^{\circ} 01^{\prime}$, 12-08-2006, 5 galls, Göllüören Village, N 40² $28^{\prime}$ E $31^{\circ}$ $35^{\prime}, 12-08-2006,3$ galls, Samsaçavuş Village, N 40² $9^{\prime}$ E $31^{\circ} 02^{\prime}, 12-08-2006,4$ galls. Host: Quercus petraea.

\section{Andricus gallaetinctoriae (Olivier, 1791)}

Material examined: Gölköy, N 40 71' E 31 51', 01-09-2011, 8 galls, Gerede Aktaşkurtlar, $\mathrm{N} 40^{\circ} 50^{\prime}$ E $32^{\circ} 31^{\prime}$, 22-08-2010, 4 galls, Macarlar Village, N 40 $49^{\prime}$ E $32^{\circ} 31^{\prime}$, 22-08-2010, 6 galls, Mengen Karakaya Village, N 40 54' E 31 ${ }^{\circ} 57^{\prime}$, 05-08-2009, 4 galls, Mudurnu, N 40 $34^{\prime}$

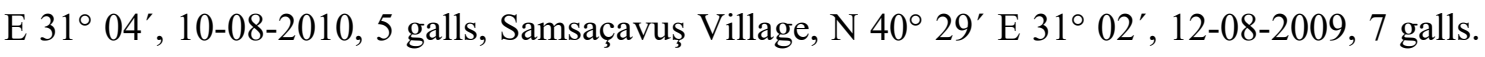
Host: Quercus petraea.

\section{Andricus glutinosus Giraud, 1859}

Material examined: Dörtdivan Çetikören, N 40 39’ E $31^{\circ}$ 59' , 17-09-2007, 4 galls, Kındıra

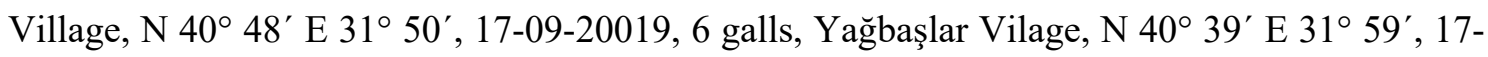

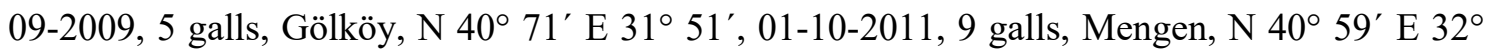
03', 14-10-2011, near Dorukhan, N 41 $00^{\prime}$ E 32 03', 05-08-2009, 1 gall, Başyellice Village, N $41^{\circ} 07^{\prime}$ E $32^{\circ} 58^{\prime}, 05-08-2009,5$ galls. Host: Quercus petraea.

\section{Andricus grossulariae Giraud, 1859}

Material examined: Mengen Babahızır, N 40 54' E 32 03', 14-05-2011, 1 gall, Gökçesu, $\mathrm{N} 41^{\circ} 26^{\prime} \mathrm{E} 32^{\circ} 24^{\prime}, 18-07-2006,1$ gall. Host: Quercus petraea, Q. robur.

\section{Andricus infectorius (Hartig, 1843)}

Material examined: Dörtdivan Çetikören, N 40 $39^{\prime}$ E $31^{\circ}$ 59', 17-09-2007, 6 galls, Kındıra

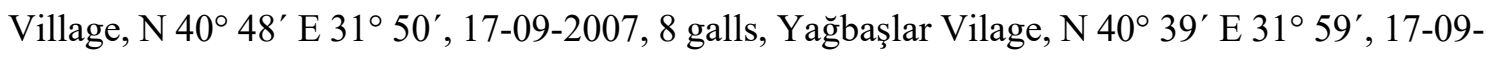

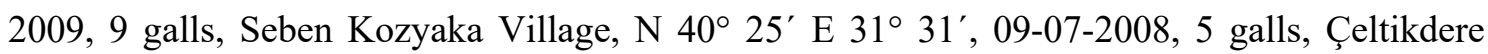
Village, N 40 $25^{\prime}$ E $31^{\circ} 31^{\prime}$, 09-07-2008, 5 galls, Gerede Dağkara Village, N 40 $19^{\prime}$ E $31^{\circ} 39^{\prime}$, 09-07-2008, 2 galls, Gölköy, N 40 71' E 31ํ 51', 19-09-2011, 5 galls, Mengen Avşar Village, N $40^{\circ} 44^{\prime}$ E $31^{\circ} 52^{\prime}, 27-07-2009,1$ gall. Host: Quercus petraea. 


\section{Andricus inflator Hartig, 1840}

Material examined: Abant, N 40 $71^{\prime}$ E $31^{\circ} 51^{\prime}$, 04-09-2011, 6 galls, Gerede Macarlar Village, N 40 $49^{\prime}$ E $32^{\circ} 31^{\prime}$, 22-09-2010, 7 galls, Salur-Karapazar, N 40 $47^{\prime}$ E $32^{\circ} 22^{\prime}$, 11-092011, 3 galls, Gölköy, N 40 71' E 31 51', 02-09-2011, 7 galls , Göynük Karacalar Village, N $40^{\circ} 22^{\prime}$ E $30^{\circ} 37^{\prime}, 10-09-2008,6$ galls, Kibriscik Belen Village, N 40 $22^{\prime}$ E $31^{\circ} 70^{\prime}, 15-09-2009$,

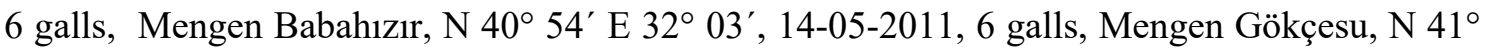

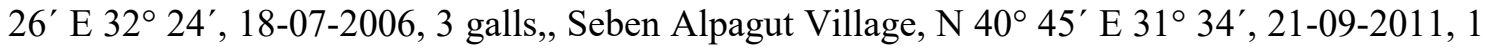
gall. Host: Quercus infectoria.

\section{Andricus kollari (Hartig, 1843)}

Material examined: Dörtdivan Çetikören, N 40 39' E 31 59', 17-09-2007, 7 galls, Gerede

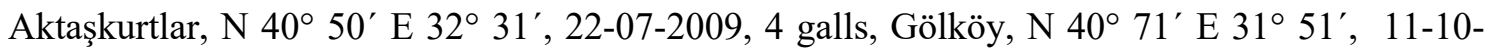
2011, 10 galls, Göynük Arıkçayırı, N 40 28' E 30 54', 19-08-2008, 9 galls, Akçaalan, N $40^{\circ}$ $28^{\prime}$ E $30^{\circ} 55^{\prime}, 05-08-2010,4$ galls Mengen, $N$ 40 59' E 32 03', 14-10-2011, 5 galls, Mudurnu Ekinören Village, N 40 $33^{\prime}$ E $31^{\circ} 01^{\prime}$, 12-08-2009, 7 galls, Gelinözü, N 40 $27^{\prime}$ E $31^{\circ} 05^{\prime}$, $12-$ 08-2009, 3 galls, Göllüören Village, N 40 $28^{\prime}$ E 31 $35^{\prime}$, 12-08-2009, 2 galls, Taşkesti, N $40^{\circ}$ $34^{\prime}$ E $31^{\circ} 04^{\prime}, 12-08-2009,5$ galls, Taşlık Village, N 40 $11^{\prime}$ E $31^{\circ} 11^{\prime}, 12-08-2009,9$ galls, Seben Kozyaka, N 40²5’ E 31 31', 09-07-2008, 8 galls. Host: Quercus petraea.

\section{Andricus lignicolus (Hartig, 1843)}

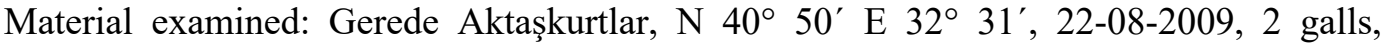

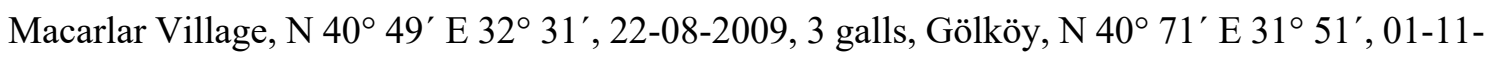
2011, 1 gall, Göynük Arıkçayırı, N 40 28' E 30 54', 19-08-2008, 6 galls, Akçaalan, N 40² $8^{\prime}$ E 30 55', 05-08-2010, 2 galls, Mengen Dereköy-Bürnük, N 40 57' E 32 08', 05-08-2010, 12 galls, Karacalar Village, N $40^{\circ} 57^{\prime}$ E $32^{\circ} 14^{\prime}$, 05-08-2010, 3 galls, Mudurnu Ekinören Village, N $40^{\circ} 33^{\prime}$ E $31^{\circ} 01^{\prime}$, 12-08-2009, 4 galls. Host: Quercus petraea, Q. infectoria, Q, robur.

\section{Andricus lucidus (Hartig, 1843)}

Material examined: Dörtdivan Çetikören, N 40 39' E 31 ${ }^{\circ}$ 59', 17-09-2007, 8 galls, Kındıra

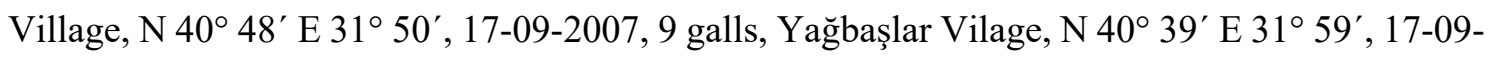
2009, 6 galls, Gölköy, N 40 71’ E 31 51', 10-11-2011, 1 gall, Göynük, Arıkçayırı, N 40²8’ E $30^{\circ} 54^{\prime}, 10-09-2008,2$ galls, Karacalar Village, N 40 $22^{\prime}$ E 30 37', 10-09-2008, 3 galls,

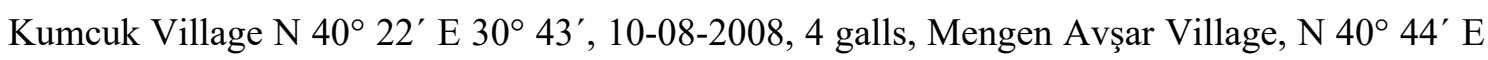
$31^{\circ} 52^{\prime}, 27-07-2009,6$ galls, Karakaya village, N 40 $54^{\prime}$ E $31^{\circ} 57^{\prime}, 25-08-2009,2$ galls. Host: Quercus petraea. 


\section{Andricus malpighii (Adler, 1881)}

Material examined: Gölköy, N 40 $71^{\prime}$ E $31^{\circ} 51^{\prime}$, 11-10-2011, 15 galls, Abant, N 40 71' E $31^{\circ} 51^{\prime}, 04-06-2011,12$ galls, Aladağ, N 40 40’ E 31 ${ }^{\circ} 38^{\prime}$, 09-07-2008, 17 galls. Host: Quercus petraea.

\section{Andricus mitratus (Mayr, 1870)}

Material examined: Aladağ, N 40 40' E 31 38', 09-09-2008, 9 galls, Dörtdivan Çetikören, $\mathrm{N} 40^{\circ} 39^{\prime} \mathrm{E} 31^{\circ} 59^{\prime}, 17-09-2007,8$ galls, Yağbaşlar Vilage, N 403' E $31^{\circ} 59^{\prime}, 27-07-2009,3$

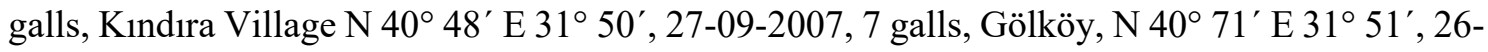

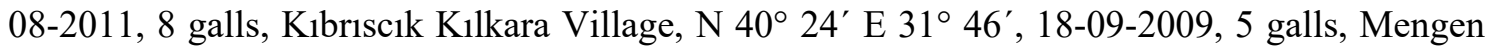
Çubuk Village, N $40^{\circ} 59^{\prime}$ E $32^{\circ} 04^{\prime}$, 14-08-2007, 9 galls, Karakaya village, N $40^{\circ} 54^{\prime}$ E $31^{\circ} 57^{\prime}$, 05-08-2009, 8 galls, Seben Çeltikdere, N 40 $22^{\prime}$ E 31 45', 15-08-2009, 13 galls, Güneyce Village, N $40^{\circ} 21^{\prime}$ E $31^{\circ} 28^{\prime}$, 09-08-2008, 6 galls. Host: Quercus petraea, Q. infectoria, $Q$. pubescens.

\section{Andricus quercusramuli (Linnaeus, 1761)}

Material examined: Abant, N 40 $71^{\prime}$ E $31^{\circ} 51^{\prime}$, 04-06-2011, 8 galls, Aladağ, N $40^{\circ} 40^{\prime} \mathrm{E}$

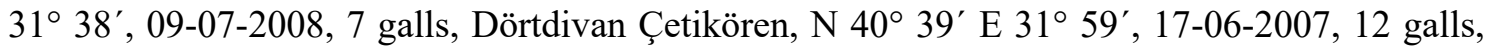

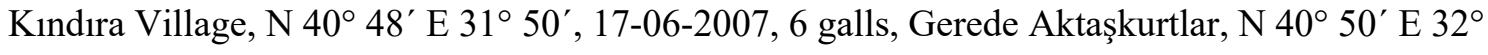
31', 22-06-2009, 5 galls, Gölköy, N 40 71' E 31 51', 14-7-2011, 21 galls, Göynük Arıkçayırı, N $40^{\circ} 28^{\prime}$ E $30^{\circ} 54^{\prime}, 10-06-2008,7$ galls, Kürnuç Village, N 40 13’ E 30 41', 17-09-2011, 9

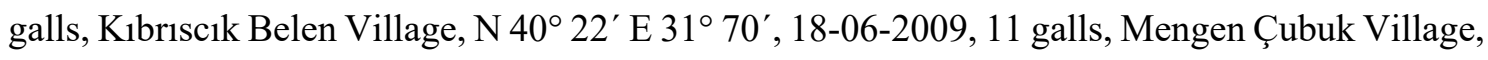
N $40^{\circ} 59^{\prime}$ E $32^{\circ} 04^{\prime}$, 14-6-2011, 4 galls, Mudurnu Ekinören Village, N 40 $33^{\prime}$ E $31^{\circ} 01^{\prime}$, 12-062009, 5 galls. Host: Quercus petraea, Q. infectoria.

\section{Andricus quercustozae (Bosc, 1792)}

Material examined: Aladağ, N 40 40' E $31^{\circ} 38^{\prime}$, 09-07-2008, 12 galls, Dörtdivan,

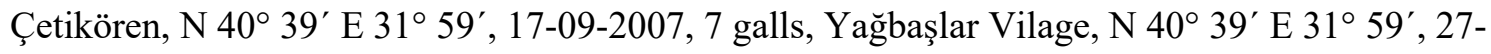
07-2009, 9 galls, Kındıra Village, N 40 48’ E 31 50', 27-07-2007, 6 galls, Gölköy, N 40 71' E $31^{\circ} 51^{\prime}$, 26-08-2011, 8 galls, Kibriscik Belen Village, N 40 22' E 31 ${ }^{\circ} 70^{\prime}, 18-09-2009,4$ galls, Kılkara Village, N 40 $24^{\prime}$ E $31^{\circ} 46^{\prime}$, 18-09-2009, 7 galls, Mengen Çubuk Village, N 40 59' E $32^{\circ} 04^{\prime}, 14-07-2007,2$ galls, Karakaya village, N 40 54' E $31^{\circ} 57^{\prime}, 05-08-2009$, 4 galls, Seben Çeltikdere, N $40^{\circ} 22^{\prime}$ E $31^{\circ} 45^{\prime}$, 15-07-2009, 3 galls, Güneyce Village, N 40 $21^{\prime}$ E $31^{\circ} 28^{\prime}, 09$ 07-2008, 9 galls, Kozyaka, N 40² $25^{\prime}$ E $31^{\circ} 31^{\prime}$, 09-07-2008, 8 galls. Host: Quercus petraea, $Q$. infectoria. 


\section{Andricus solitarius (Fonscolombe, 1832)}

Material examined: Mengen Gökçesu, N 41ํ $21^{\prime}$ E $32^{\circ} 10^{\prime}$, 18-07-2006, 5 galls. Host: Quercus petraea.

\section{Andricus stefanii (Kieffer, 1897)}

Material examined: Mengen Çubuk Village, N 40 59' E 32 04', 14-10-2011, 2 galls, Gölköy, N 40 $71^{\prime}$ E 31 51', 09-10-2011, 5 galls, Seben Kozyaka N 40 25' E 31 31', 09-072008, 1 gall, Yeniçağ Saray-Yamanlar, N 40 47’ E 32²2', 11-06-2011, 2 galls. Host: Quercus petraea.

\section{Andricus sternlichti Bellido, Pujade-Villar, Melika, 2003}

Material examined: Gölköy, N 40 71' E 31 51', 09-10-2011, 10 galls, Hamidiye area, N $40^{\circ} 67^{\prime}$ E $31^{\circ} 58^{\prime}, 29-10-2010,13$ galls, Kibrisc1k Belen Village, N 40 22' E 31 70', 18-092009, 7 galls, K1lkara Village, N 40² $4^{\prime}$ E $31^{\circ} 46^{\prime}$, 18-09-2009, 2 galls, Mudurnu Nallıhan Road, N $40^{\circ} 28^{\prime}$ E $31^{\circ} 11^{\prime}$, 12-06-2009, 8 galls. Host: Quercus petraea, Q. pubescens.

\section{Andricus theophrastea (Trotter, 1866)}

Material examined: Gölköy, N 40 $71^{\prime}$ E $31^{\circ}$ 51', 09-10-2011, 5 galls, Hamidiye area, N

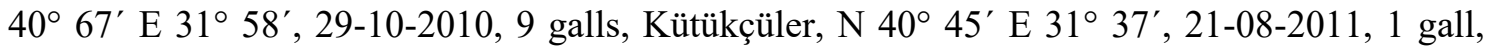

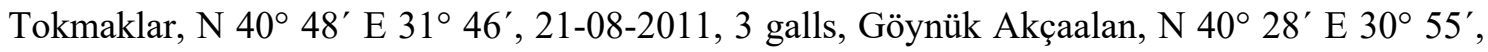
05-08-2010, 2 galls, Arıkçayırı, N 40 28' E 30 54', 19-08-2008, 4 galls, Bulanık Village, N $40^{\circ}$ $24^{\prime}$ E $30^{\circ} 46^{\prime}, 19-08-2010,1$ gall, Karacalar Village, N 40² $22^{\prime}$ E $30^{\circ} 37^{\prime}, 10-09-2008,2$ galls, Kumcuk Village, N 40²2' E 30 43', 19-08-2010, 3 galls. Host: Quercus petraea.

\section{Andricus tomentosus (Trotter, 1901)}

Material examined: Hamidiye area, N $40^{\circ} 67^{\prime}$ E $31^{\circ} 58^{\prime}, 21-09-2009,15$ galls. Host: Quercus petraea.

\section{Biorhiza pallida (Olivier, 1791)}

Material examined: Gerede Aktaşkurtlar, N 40 50' E 32 $31^{\circ}$, 22-06-2010, 5 galls,

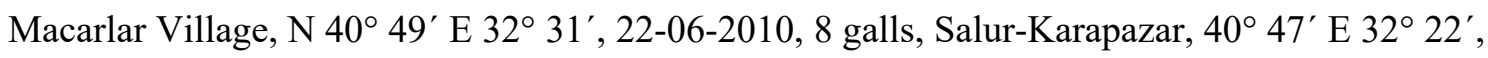
11-06-2011, 7 galls, Gölköy, N 40 71' E 31 51', 02-06-2011, 14 galls, Göynük Akçaalan, N $40^{\circ} 28^{\prime}$ E $30^{\circ} 55^{\prime}, 05-06-2010,9$ galls, Arıkçayırı, N 40 28' E 30 54', 19-06-2008, 8 galls, Karacalar Village, $\mathrm{N} 40^{\circ} 22^{\prime}$ E $30^{\circ} 37^{\prime}$, 10-06-2008, 6 galls, Kibriscik Belen Village, N $40^{\circ} 22^{\prime}$ 
E $31^{\circ} 70^{\prime}, 15-06-2009,9$ galls, Mengen Babahızır, N 40 54' E 32 03', 14-06-2011, 7 galls,

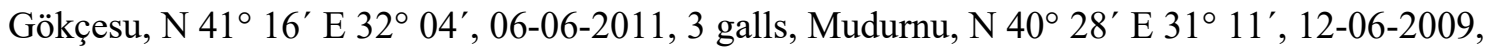
2 galls, Seben Alpagut Village, N 40 45’ E 31 ${ }^{\circ} 34^{\prime}, 21-06-2011,2$ galls. Host: Quercus petraea, Q. infectoria.

\section{Cerroneuroterus lanuginosus (Giraud, 1859)}

Material examined: Aladağ, N 40 40' E $31^{\circ} 38^{\prime}$, 09-07-2008, 13 galls, Abant, N 40 $71^{\prime}$ E $31^{\circ} 51^{\prime}, 04-09-2011,21$ galls, Mengen Çubuk Village, N 40 59' E 32 ${ }^{\circ} 04^{\prime}, 14-07-2007,8$ galls. Host: Quercus petraea.

\section{Cerroneuroterus minutulus (Giraud, 1859)}

Material examined: Abant, N 40 71' E 31 ${ }^{\circ}$ 51', 04-09-2011, 15 galls, Gölköy, N 40 71' E $31^{\circ}$ 51', 26-08-2011, 7 galls, Kibriscik Belen Village, N 40² 22' E 31 70', 18-09-2009, 3 galls, Kilkara Village, N 40 24' E $31^{\circ} 46^{\prime}, 18-09-2009$, 4 galls, Mengen Çubuk Village, N 40 $59^{\prime} \mathrm{E}$ $32^{\circ} 04^{\prime}$, 14-07-2007, 6 galls, Karakaya village, N 40 54' E $31^{\circ} 57^{\prime}, 05-08-2009$, 5 galls, Seben Çeltikdere, N $40^{\circ} 22^{\prime}$ E $31^{\circ} 45^{\prime}$, 15-07-2009, 2 galls, Güneyce Village, N 40 $21^{\prime}$ E $31^{\circ} 28^{\prime}, 09$ 07-2008, 2 galls, Kozyaka, N 40² $25^{\prime}$ E $31^{\circ} 31^{\prime}$, 09-07-2008, 7 galls. Host: Quercus petraea, $Q$. infectoria.

\section{Chilaspis nitida (Giraud, 1859)}

Material examined: Abant, N 40 $71^{\prime}$ E $31^{\circ} 51^{\prime}$, 04-09-2011, 10 galls, Gölköy, N 40 71' E $31^{\circ}$ 51', 26-08-2011, 8 galls. Host: Quercus petraea.

\section{Cynips agama Hartig, 1840}

Material examined: Aladağ, N 40 40’ E 31 38', 09-07-2008, 5 galls, Dörtdivan Çetikören, N 40 39' E $31^{\circ} 59^{\prime}, 17-09-2007,8$ galls, Kindira Village, N 40 $48^{\prime}$ E $31^{\circ} 50^{\prime}, 27-07-2007,3$ galls, Gölköy, N 40 $71^{\prime}$ E $31^{\circ}$ 51', 26-08-2011, 6 galls, Kibrıscık Kılkara Village, N 40² $24^{\prime}$ E $31^{\circ} 46^{\prime}, 18-09-2009,4$ galls, Mengen, Çubuk Village, N 40 59' E 32 04', 14-07-2007, 7 galls, Seben Çeltikdere, N $40^{\circ} 22^{\prime}$ E $31^{\circ} 45^{\prime}, 15-07-2009,5$ galls, Güneyce Village, N 40 $21^{\prime}$ E $31^{\circ}$ 28', 09-07-2008, 2 galls, Kozyaka N 40 25' E 31 $31^{\prime}$, 09-07-2008, 4 galls. Host: Quercus petraea, Q. infectoria.

\section{Cynips disticha Hartig, 1840}

Material examined: Abant, N 40 $71^{\prime}$ E $31^{\circ}$ 51', 04-09-2011, 13 galls, Gerede Aktaşkurtlar, $\mathrm{N} 40^{\circ} 50^{\prime}$ E $32^{\circ} 31^{\prime}, 22-09-2009,8$ galls, Ertuğrulköy Village, N 40 49’ E 32 21', 11-09-2011, 
7 galls, Gölköy, N 40 $71^{\prime}$ E $31^{\circ}$ 51', 26-05-2011, 16 galls, Mengen, N $40^{\circ} 59^{\prime}$ E $32^{\circ}$ 03', 14-102011, Mudurnu Güllüören Village, N 40² 28' E 31 35', 14-10-2011 3 galls, Taşkesti, N 40 34' E $31^{\circ} 04^{\prime}, 12-08-2009,11$ galls, near Taşlik Village, $N 40^{\circ} 11^{\prime}$ E $31^{\circ} 11^{\prime}, 14-10-2011,5$ galls. Host: Quercus petraea, Q. pubescens.

\section{Cynips divisa Hartig, 1840}

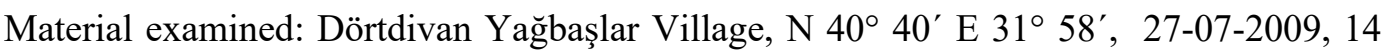
galls, Gerede Aktaşkurtlar, N 40 50' E 32॰ 31', 22-07-2009, 21 galls, Macarlar Village, N $40^{\circ}$

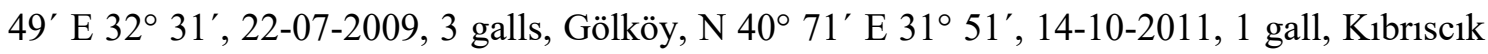
Kilkara Village, N 40 $24^{\prime}$ E $31^{\circ} 46^{\prime}, 15-07-2009,3$ galls, near Belen Village, N 40 $25^{\prime}$ E $31^{\circ}$ 45', 17-07-2009, 3 galls, Hamidiye Village, N 40 73' E $31^{\circ} 54^{\prime}, 14-10-2011,7$ galls. Host: Quercus petraea.

\section{Cynips longiventris Hartig, 1840}

Material examined: Abant, N 40 $71^{\prime}$ E $31^{\circ} 51^{\prime}$, 04-09-2011, 1 gall, Gerede, N 40 $40^{\prime} \mathrm{E}$

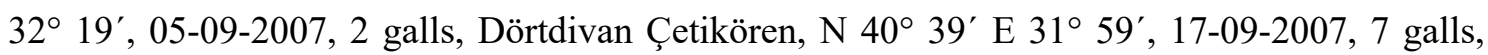
Kındira Village, N 40 48' E 31 50', 17-09-2007, 9 galls, Gerede Aktaşkurtlar, N 40 50' E 32 ${ }^{\circ}$ 31', 22-09-2009, 7 galls, Macarlar Village, N 40 49' E 32 31', 22-09-2009, 6 galls, Gölköy, N $40^{\circ} 71^{\prime}$ E $31^{\circ} 51^{\prime}$, 02-06-2011, 20 galls, Kibriscik Kilkara Village, N 40 $24^{\prime}$ E 31 $46^{\prime}$, 18-09-

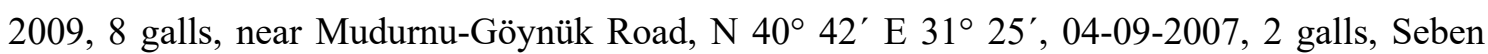
Çeltikdere, N $40^{\circ} 22^{\prime}$ E $31^{\circ} 45^{\prime}$, 15-08-2008, 3 galls. Host: Quercus petraea, Q. infectoria.

\section{Cynips quercus (Fourcroy, 1785)}

Material examined: Abant, N 40 $71^{\prime}$ E $31^{\circ}$ 51', 04-09-2011, 17 galls, Göynük Akçaalan Village, N 40 $28^{\prime}$ E $30^{\circ} 55^{\prime}$, 19-08-2007, 11 galls, Arıkçayırı Village, N 40 28' E 30 54', 1908-2007, 10 galls, Göynük Kürnuç Village, N 40 13’ E 30 41', 19-08-2007, 7 galls, Kumcuk

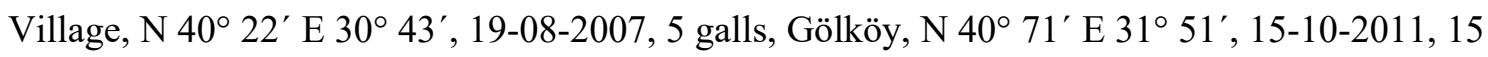
galls. Host: Quercus petraea.

\section{Cynips quercusfolii (Linnaeus, 1758)}

Material examined: Abant, N 40 $71^{\prime}$ E $31^{\circ} 51^{\prime}$, 04-09-2011, 8 galls, Aladağ, N 40 40' E

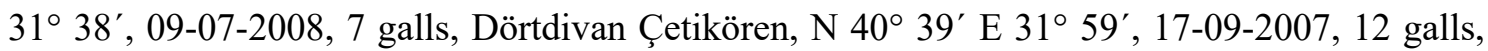

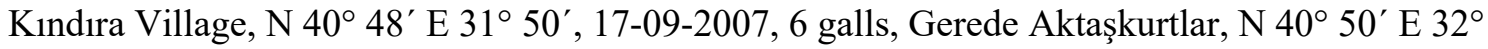
31', 22-07-2009, 5 galls, Gölköy, N 40 71' E 31 51', 14-10-2011, 21 galls, Göynük Arıkçayırı, $\mathrm{N} 40^{\circ} 28^{\prime}$ E $30^{\circ} 54^{\prime}, 10-09-2008,7$ galls, Bulanık Village, N 40² $24^{\prime}$ E $30^{\circ} 46^{\prime}, 15-09-2010,2$ 


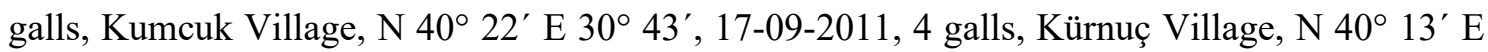
$30^{\circ} 41^{\prime}$, 17-09-2011, 9 galls, Kibriscik Belen Village, N 40 22' E 31 70', 18-09-2009, 11 galls, Kılkara Village, N 40² $24^{\prime}$ E $31^{\circ} 46^{\prime}, 18-09-2009,7$ galls, Mengen Çubuk Village, N 40 59’ E $32^{\circ} 04^{\prime}, 14-10-2011,4$ galls, Karakaya village, N $40^{\circ} 54^{\prime}$ E $31^{\circ} 57^{\prime}, 25-08-2009,1$ gall, Mudurnu

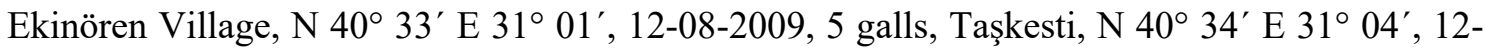

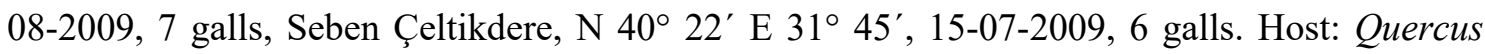
petraea, Q. infectoria.

\section{Neuroterus albipes (Schenck, 1863)}

Material examined: Abant, N 40 $71^{\prime}$ E $31^{\circ}$ 51', 04-09-2011, 21 galls. Host: Quercus petraea.

\section{Neuroterus anthracinus (Curtis, 1838)}

Material examined: Aladağ, N 40 40' E 31 $38^{\prime}$, 09-07-2008, 8 galls, Abant, N 40 $71^{\prime} \mathrm{E}$ $31^{\circ}$ 51', 04-09-2011, 12 galls, Dörtdivan Çetikören, N 40 39' E 31 59', 17-09-2007, 4 galls,

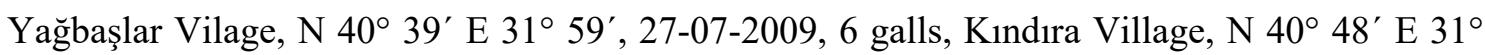
50', 27-07-2007, 3 galls, Gölköy, N 40 71' E 31 51', 14-10-2011, 1 gall, Mengen Çubuk Village, N $40^{\circ} 59^{\prime}$ E $32^{\circ} 04^{\prime}, 14-07-2007$, 4 galls, Karakaya village, N $40^{\circ} 54^{\prime}$ E $31^{\circ} 57^{\prime}$, 05-082009, 6 galls. Host: Quercus petraea.

\section{Neuroterus numismalis (Fourcroy, 1785)}

Material examined: Abant, N 40 $71^{\prime}$ E $31^{\circ}$ 51', 04-09-2011, 15 galls, Dörtdivan Çetikören, N 40 39' E $31^{\circ} 59^{\prime}, 17-09-2007,2$ galls, Kındıra Village, N 40 48' E $31^{\circ} 50^{\prime}$, 17-09-2007, 7 galls, Göynük, Arıkçayırı, N 40 28' E 30 54', 10-09-2008, 11 galls, Bulanık Village, N 40 24' E $30^{\circ} 46^{\prime}, 15-09-2010,8$ galls, Mengen, N 40 59’ E 32 03', 14-10-2011, 12 galls, Gökçesu, N $41^{\circ} 16^{\prime}$ E $32^{\circ} 04^{\prime}$, 06-06-2011, 6 galls, Seben near Alpagut Village, N 40 45’ E 31 ${ }^{\circ} 34^{\prime}, 21-08-$ 2011, 3 galls. Host: Quercus petraea, Q. pubescens.

\section{Neuroterus politus Hartig, 1840}

Material examined: Abant, N 40 $71^{\prime}$ E $31^{\circ} 51^{\prime}$, 04-05-2011, 6 galls, Gerede Macarlar

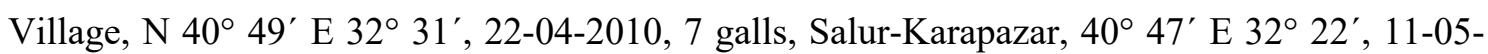
2011, 3 galls, Gölköy, N 40 71' E 31 51', 02-05-2011, 7 galls, Göynük Arıkçayırı, N 40² 28’ E $30^{\circ} 54^{\prime}, 19-04-2008,3$ galls, Karacalar Village, N 40 $22^{\prime}$ E $30^{\circ} 37^{\prime}, 10-05-2008,6$ galls,

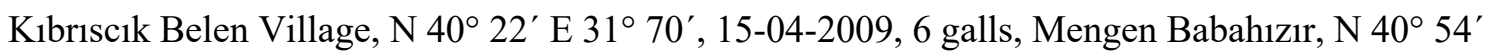


E $32^{\circ} 03^{\prime}, 14-05-2011,6$ galls, Mudurnu Nallihan Road, N 40 $28^{\prime}$ E $31^{\circ} 11^{\prime}, 12-05-2009,3$ galls, Seben Alpagut Village, N 40 45’ E 31 34', 21-04-2011, 1 gall. Host: Quercus petraea.

\section{Neuroterus quercusbaccarum (Linnaeus, 1758)}

Material examined: Abant, N 40 $71^{\prime}$ E $31^{\circ}$ 51', 04-09-2011, 9 galls, Dörtdivan Çetikören,

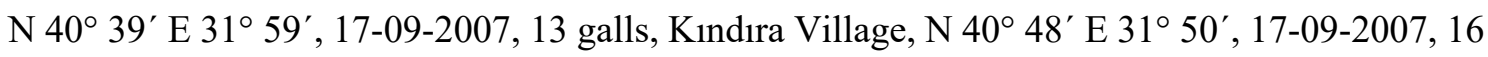
galls, Gölköy, N 40 $71^{\prime}$ E $31^{\circ} 51^{\prime}$, 03-06-2011, 2 galls, Kıbrıscık Belen Village, N 40² $22^{\prime}$ E $31^{\circ}$ 70', 18-09-2009, 5 galls, K1lkara Village, N 40² 24' E 31 46', 18-09-2009, 8 galls, Mengen Avşar Village, N 40 86’ E $31^{\circ} 84^{\prime}$, 06-06-2011, 10 galls, Gökçesu, N $41^{\circ} 16^{\prime}$ E 32 ${ }^{\circ} 04^{\prime}$, 06-062011, 4 galls, Mudurnu Ekinören Village, N 40 33' E 31 ${ }^{\circ}$ 01', 12-08-2009, 15 galls, Taşkesti, N $40^{\circ} 34^{\prime}$ E $31^{\circ} 04^{\prime}, 12-08-2009,17$ galls, Seben Çeltikdere, N 40 $22^{\prime}$ E $31^{\circ} 45^{\prime}, 15-07-2009,16$ galls. Host: Quercus petraea, Q. pubescens.

\section{Neuroterus tricolor (Hartig, 1841)}

Material examined: Abant, N 40 71' E 31 51', 04-09-2011, 22 galls, Dörtdivan Çetikören, N $40^{\circ} 39^{\prime}$ E $31^{\circ} 59^{\prime}, 17-09-2007,11$ galls, Kindıra Village, N 40 $48^{\prime}$ E $31^{\circ} 50^{\prime}, 17-09-2007,8$ gall, Yağbaşlar Vilage, N 40 39’ E 31 59', 17-09-2009, 7 galls, Gölköy, N 40 71' E $31^{\circ} 51^{\prime}$, 10-11-2011, 2 galls, Göynük, Arıkçayırı, N 40²8' E 30 54', 10-09-2008, 3 galls, Karacalar Village, N 40 $22^{\prime}$ E $30^{\circ} 37^{\prime}$, 10-09-2008, 3 galls, Mengen Avşar Village, N 40 44' E $31^{\circ} 52^{\prime}$, 27-07-2009, 10 galls. Host: Quercus petraea, Q. infectoria.

\section{Pseudoneuroterus saliens (Kollar, 1857)}

Material examined: Abant, N 40 71' E 31 ${ }^{\circ}$ 51', 04-09-2011, 3 galls, Gölköy, N 40 $71^{\prime}$ E $31^{\circ} 51^{\prime}$, 10-11-2011, 2 galls, Dörtdivan Kındıra Village, N 40 48’ E 31 $50^{\circ}$, 17-09-2007, 1 gall, Göynük Karacalar Village, N $40^{\circ} 22^{\prime}$ E $30^{\circ} 37^{\prime}$, 10-09-2008, 2 galls, Seben Çeltikdere, N $40^{\circ}$ $22^{\prime}$ E $31^{\circ} 45^{\prime}$, 15-07-2009, 2 galls. Host: Quercus cerris, Q. petraea, Q. infectoria.

\section{Trigonaspis synaspis Hartig, 1841}

Material examined: Abant, N 40 $71^{\prime}$ E $31^{\circ} 51^{\prime}$, 04-09-2011, 9 galls, Gerede, N $40^{\circ} 40^{\prime} \mathrm{E}$ $32^{\circ} 19^{\prime}$, 05-09-2007, 4 galls, Dörtdivan Çetikören, N 40³9' E 31 59', 17-09-2007, 5 galls,

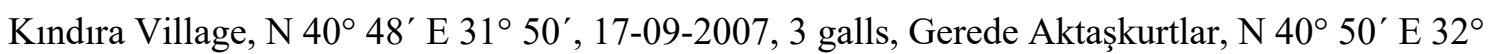
31', 22-09-2009, 6 galls, Macarlar Village, N 40 49' E 32 31', 22-09-2009, 6 galls, Gölköy, N $40^{\circ} 71^{\prime}$ E $31^{\circ} 51^{\prime}, 14-105-2011,7$ galls, Kibriscik Kilkara Village, N 40 $24^{\prime}$ E $31^{\circ} 46^{\prime}$, 18-092009, 3 galls, Seben Çeltikdere, N 40² $22^{\prime}$ E $31^{\circ} 45^{\prime}$, 15-08-2008, 2 galls. Host: Quercus petraea, Q. pubescens. 
With this study, we found 44 cynipid species belonging to 8 different genera from the tribe Cynipini in Bolu. We determined that Andricus genus is the most specious group in Bolu which is represented by 26 species. The other genera members in the studied area include Biorhiza with one species, Cerroneuroterus with 2 species, Chilaspis with one species, Cynips and Neuroterus with 6 species each, Pesudoneuroterus and Trigonaspis genera are with one species.

\section{Conclusions}

This is the first detailed work on oak cynipids found in Bolu where all the given cynipid species in this study are recorded for the first time from the studied area. Latest studies on this parasitic insect group of oaks are becoming more popular and requires more attention. Reviewing the literature, it seems the cynipid fauna of Turkey has not yet been completed and requires further detailed investigation.

\section{References}

[1] Ronquist, F., Liljeblad, J., Evolution of the gall wasp-host plant association, Evolution, 55, 2503-2522, 2001.

[2] Stone, G.N., Cook, J.M., The structure of cynipid oak galls: patterns in the evolution of an extended phenotype, Proceedings of the Royal Society of London B, 265, 979-988, 1998.

[3] Stone, G.N., Schönrogge, R.A., Atkinson, K., Bellido, D., Pujade-Villar, J., The population biology of oak gallwasps (Hymenoptera: Cynipidae), Annual Review of Entomology 47, 633-668, 2002.

[4] Abe, Y., Melika, G., Stone, G.N., The diversity and phylogeography of cynipid gallwasps (Hymenoptera: Cynipidae) of the Oriental and Eastern Palearctic Regions, and their associated communities, Oriental Insects, 41, 169-212, 2007.

[5] Oğurlu, İ., Avc1, M., "Kasnak meşesi Quercus vulcanica (Boiss. and Held.) Kotschy' da zarar yapan böcekler," in Kasnak Meşesi ve Türkiye Florası Sempozyumu, pp. 657-671, 1998.

[6] Melika, G., Gallwasps of Ukraine: Cynipidae. Schmalhausen Institute of Zoology, National Academy of Sciences of Ukraine, 646 pp (2 vols), 2006.

[7[ Yılmaz, H., Quercus L. (Ed. Ü. Akkemik), Türkiye'nin Doğal-Egzotik Ağaç ve Çalıları. Ankara: Orman Genel Müdürlüğ̈̈ Yayınları, 338-356, 2018.

[8] OGM (Orman Genel Müdürlüğ̈̈ / General Directorate of Forestry). (2019, November 11). Ormancllık İstatistikleri. https://www.ogm.gov.tr/ ekutuphane/Sayfalar/Istatistikler.aspx.

[9] Melika, G., Stone, G.N., A new species of cynipid gall wasp from Turkey (Hymenoptera: Cynipidae), Folia Entemolica Hungarica, 62, 127-131, 2001.

[10] Kıyak, S., Kılıç, T., Katılmış, Y., A contribution to the knowledge of the Cynipini fauna of Turkey (Hymenoptera: Cynipidae), Munis Entomology \& Zoology, 3(1), 523-536, 2008.

[11] Katılmış, Y., Kıyak, S., Checklist of Cynipidae of Turkey, with a New Genus Record, Journal of Natural History, 42(31-32), 2161-2167, 2008. 
[12] Katılmış, Y., Kıyak, S., New records of oak gallwasps of the genus Andricus (Burgsdorf, 1783) from Turkey (Hymenoptera: Cynipidae), Zoology in the Middle East, 48, 108109, 2009.

[13] Katılmış Y., Kıyak, S., Oak gallwasps (Hymenoptera: Cynipidae) fauna of innerwestern Anatolian, Munis Entomology \& Zoology, 6(2), 735-757, 2011.

[14] Mutun, S., Dinç, S., Contribution of the Turkish Cynipidae fauna with one new record, Journal of Applied Biological Sciences, 5(3), 83-85, 2011.

[15] Mete, Ö., Demirsoy, A., Kemaliye'nin (Erzincan, Türkiye) gal arısı faunası üzerine bir ön çalışma ve Türkiye için yeni bir kayıt, Hacettepe Journal of Biology \& Chemistry, 351$363,2012$.

[16] Dinç, S., Mutun, S., Melika, G., A new species of Andricus Hartig Oak Gall Wasp from Turkey (Hymenoptera: Cynipidae, Cynipini), North-Western Journal of Zoology, 10(1), 122-127, 2014.

[17] Mutun, S., Dinç, S., Bozsó, M., Melika, G., Four New Species of Andricus Hartig Oak Gallwasp From Turkey (Hymenoptera: Cynipidae, Cynipini), Zootaxa, 3760(2), 241-259, 2014.

[18] Katılmış, Y., Azmaz, M., Investigation on the inquilines (Hymenoptera: Cynipidae, Synergini) of oak galls from Inner-Western Anatolia, Turkey, Turkish Journal of Zoology, 39(1), 168-173, 2015.

[19] Azmaz, M., Katılmış, Y., Updated species list of Cynipidae (Hymenoptera) from Turkey, Zootaxa. 4303(3), 361-378, 2017.

[20] Bayrak, S., Avc1, M., Gall forming Cynipini (Hymenoptera: Cynipidae) species in Isparta oak forests, Munis Entomology \& Zoology, 14(2), 552-564, 2019. 